\title{
Tnfaip8 is an essential gene for the regulation of glucocorticoid-mediated apoptosis of thymocytes
}

\author{
MJ Woodward ${ }^{1,4}$, J de Boer ${ }^{1,4}$, S Heidorn ${ }^{1}$, M Hubank ${ }^{1}$, Kioussis $^{2}$, O Williams ${ }^{1}$ and HJM Brady ${ }^{\star, 1,3}$
}

Glucocorticoids have significant immunoregulatory actions on thymocytes and $\mathrm{T}$ cells and act by binding and activating cytosolic glucocorticoid receptors, which translocate to the nucleus and control gene expression through binding to specific response elements in target genes. Glucocorticoids promote cell death by activating an apoptotic program that requires transcriptional regulation. We set out to identify genes that are crucial to the process of glucocorticoid-mediated thymocyte apoptosis. Freshly isolated murine primary thymocytes were treated with dexamethasone, mRNA isolated and used to screen DNA microarrays. A set of candidate genes with upregulated expression was identified and selected members assayed in reconstituted fetal thymic organ culture (FTOC). Fetal liver-derived hematopoietic progenitor cells (HPCs) were infected with retroviruses expressing individual genes then used to repopulate depleted fetal thymic lobes. Reconstituted FTOCs expressing the gene Tnfaip8 were treated with dexamethasone and shown to be greatly sensitized to dexamethasone. Retrovirus-mediated RNA interference was applied to knock down Tnfaip8 expression in HPCs and these were used to reconstitute FTOCs. We observed that downregulating the expression of Tnfaip8 alone was sufficient to effectively protect thymocytes against glucocorticoid-induced apoptosis. We propose that Tnfaip8 is crucial in regulating glucocorticoid-mediated apoptosis of thymocytes.

Cell Death and Differentiation (2010) 17, 316-323; doi:10.1038/cdd.2009.125; published online 4 September 2009

Glucocorticoids have been reported to have significant immunoregulatory actions on thymocytes and T cells. ${ }^{1-3}$ They act by binding and activating cytosolic glucocorticoid receptors, which translocate to the nucleus and control gene expression by binding to glucocorticoid response elements in target genes. Glucocorticoids can promote cell death by activating an apoptotic program that requires transcriptional regulation. The inhibition of either transcription or translation will block glucocorticoid-induced apoptosis of thymocytes, indicating that de novo synthesis of mRNA and protein is obligatory. ${ }^{4-6}$ Multiple signaling pathways appear to act on the initiation of glucocorticoid-induced apoptosis. ${ }^{7}$ BCL-2 family members have an important role: for example, the thymocytes of mice null for both the Bax and Bak genes are resistant to glucocorticoid-induced apoptosis. ${ }^{8}$ Glucocorticoid-induced apoptosis in thymocytes appears to act via BAX/BAK-mediated release of cytochrome $c$ from mitochondria and activation of CASPASE-9. ${ }^{9}$ In addition, expression of BIM, a BH3-domain-only member of the BCL-2 family, has been shown to be induced by glucocorticoids. ${ }^{10}$ Thymocytes from $\mathrm{Bim}^{-1-}$ mice show significantly reduced sensitivity to glucocorticoid-induced apoptosis ${ }^{11}$ and downregulation of Bim expression in a B-cell line by RNA interference (RNAi) greatly reduces sensitivity to glucocorticoid. ${ }^{12}$

There is considerable debate regarding how endogenous glucocorticoid levels can influence thymic development and homeostasis. ${ }^{1-3,13,14}$ Glucocorticoids are produced within the thymus by thymic epithelial cells, ${ }^{15,16}$ and evidence exists to suggest they have a considerable effect not only on thymus homeostasis, but also on the nature of the developing T-cell receptor repertoire. ${ }^{2,3}$

We set out to identify genes that have a functional role in the process of glucocorticoid-mediated thymocyte apoptosis. We treated freshly isolated murine primary thymocytes with dexamethasone, isolated RNA and used this to screen DNA microarrays. A set of candidate genes with upregulated expression was identified and selected members of this set were assayed in reconstituted fetal thymic organ culture (FTOC). Fetal liver-derived hematopoietic progenitor cells (HPCs) were infected with retroviruses expressing individual candidate genes and used to repopulate depleted fetal thymic lobes. The resultant gene-specific reconstituted FTOCs were treated with dexamethasone and their sensitivity was measured. The gene Tnfaip 8 was shown to greatly sensitize thymocytes to dexamethasone in FTOCs. We then knocked down Tnfaip8 expression in HPCs using gene-specific retrovirus-mediated shRNAmiRs and used these HPCs to reconstitute FTOCs. We observed that downregulating Tnfaip8 expression alone was sufficient to effectively protect thymocytes against glucocorticoid-induced apoptosis.

\footnotetext{
${ }^{1}$ Molecular Haematology and Cancer Biology Unit, University College London Institute of Child Health, London, UK; ${ }^{2}$ Division of Molecular Immunology, MRC National Institute for Medical Research, Mill Hill, London, UK and ${ }^{3}$ Immunology and Infection Section, Division of Cell and Molecular Biology, Sir Alexander Fleming Building, Imperial College, London SW7 2AZ, UK

*Corresponding author: HJM Brady, Immunology and Infection Section, Division of Cell and Molecular Biology, Sir Alexander Fleming Building, Imperial College, London SW7 2AZ, UK. Tel: + 442075943 777; Fax: + 442075943 777; E-mail: h.brady@imperial.ac.uk

${ }^{4}$ These authors contributed equally to this work.

Keywords: apoptosis; glucocorticoid; Tnfaip8; fetal thymic organ culture; RNA interference

Abbreviations: FTOC, fetal thymic organ culture; HPC, hematopoietic progenitor culture; 2dGuo, 2-deoxyguanosine; DED, death effector domain

Received 21.10.08; revised 16.7.09; accepted 23.7.09; Edited by SJ Martin; published online 04.9.09
} 


\section{Results}

Identification of candidate glucocorticoid-regulated target genes. Our initial experiments set out to identify candidate target genes that might have a functional role in regulating glucocorticoid-mediated thymocyte apoptosis. To isolate RNA for gene array expression analysis, primary thymocytes were taken from $\mathrm{C} 57 \mathrm{BI} / 6$ mice and cultured in vitro for $4 \mathrm{~h} \pm 5 \mu \mathrm{M}$ dexamethasone $\pm 25 \mu \mathrm{M}$ roscovitine. Roscovitine is an inhibitor of cyclin-dependent kinases, which has been shown previously to delay glucocorticoid-induced thymocyte apoptosis at an early stage ${ }^{17}$ and hence allow gene expression to be analyzed following initiation of the glucocorticoid apoptotic response but before loss of mitochondrial integrity. Roscovitine blocked cell death for at least $4 \mathrm{~h}$ following exposure to dexamethasone when comparing dexamethasone + roscovitine with dexamethasone alone (Figure 1a). Thymocytes from three independent experiments were harvested $4 \mathrm{~h}$ post-treatment and RNA was isolated and processed for analysis on Affymetrix MGu74Av2 gene arrays. The genes most highly upregulated by dexamethasone in the presence of roscovitine, compared with thymocytes incubated with roscovitine alone, are detailed in Figure $1 \mathrm{~b}$ and selected members of this set were further analyzed for their role in thymocyte apoptosis.
Functional analysis of candidate genes in FTOCs and on OP9-DL1 stromal cells. Many of the dexamethasoneinduced genes found in this study have been previously reported in similar screens, including Tdag8, ${ }^{18,19}$ Fkbp51, $^{19}$ $\operatorname{Dig}^{20}$ and Bim. ${ }^{19,20}$ Overexpression of these genes changes susceptibility to apoptosis and also changes sensitivity to dexamethasone in a variety of systems. We, therefore, first addressed if the genes identified in this screen could regulate the survival of developing thymocytes. We used a retroviral expression vector that allows coexpression of individual candidate genes with a truncated version of human CD2 in HPCs (Supplementary Figure S1a). The retrovirally transduced cells can be differentiated using the FTOC system into thymocytes expressing both CD4 and CD8, so-called double positive (DP) cells. DP thymocytes are by far the most sensitive $T$ cells to glucocorticoid-mediated apoptosis. ${ }^{1}$ A mixture of human $\mathrm{CD}^{-}$(hCD2) (uninfected) and $\mathrm{hCD}^{+}$(infected) cells are used for the T-cell development assays, thereby providing an internal control of 'wild type' cells, as this allows for comparison of T-cell development within the same assay as illustrated for FTOCs in Figure 1c. Overexpression of the identified genes may impose a positive or negative survival effect on the $\mathrm{hCD} 2^{+}$ cells. The effect can be quantified when comparing the ratios between hCD2 expressing cells pre- and post-FTOC. Following co-staining for CD4 and CD8, the proportion of a
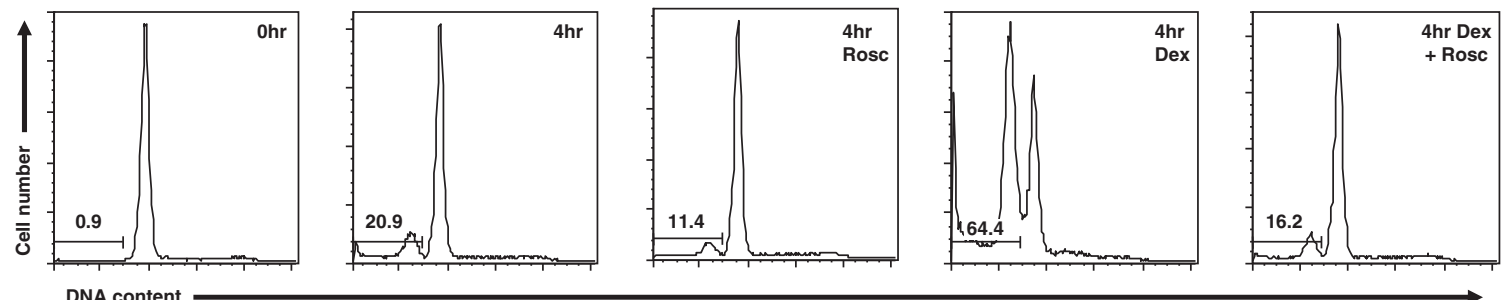

DNA content b

\begin{tabular}{lll}
\hline Genes Induced & Accession & Fold Change \\
\hline MTV Locus 43 & M90535 & 87.7 \\
E4BP4 & NM_017373 & 21.2 \\
\hline TDAG8 & NM_008152 & 16.6 \\
\hline FGFR1(L) & AK034295 & 14.2 \\
\hline TNFAIP8 & Al839109 & 8.0 \\
DIG2 & Al849939 & 7.3 \\
\hline PIP49 & Al841484 & 7.2 \\
\hline mSpp1 & Al835784 & 6.9 \\
LRG-47 & NM_008326 & 6.3 \\
Lisch7 & NM_017405 & 6.0 \\
\hline Ikaros & AK077815 & 4.6 \\
BimEL & NM_207680 & 4.3 \\
FKBP51 & NM_010220 & 4.1 \\
TNFAIP3(A20) & NM_009397 & 4.1
\end{tabular}

C

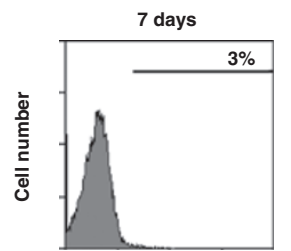

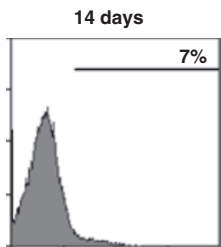
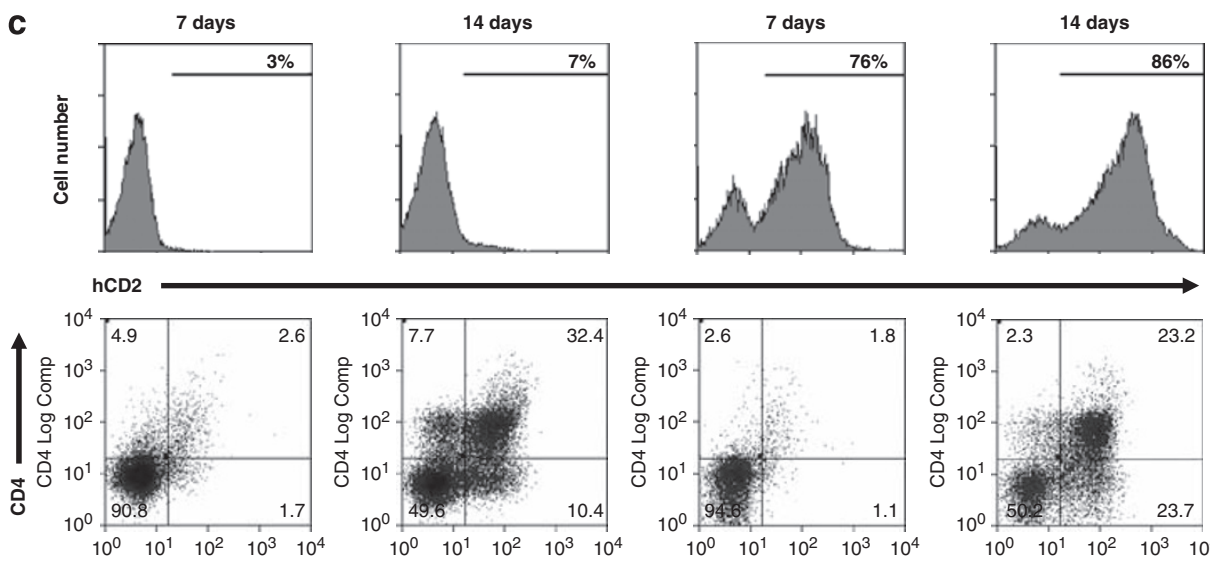

CD8 Log Comp

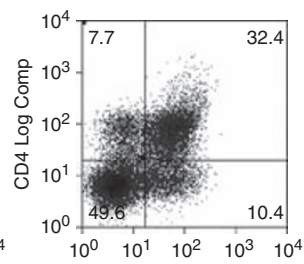

CD8 Log Comp

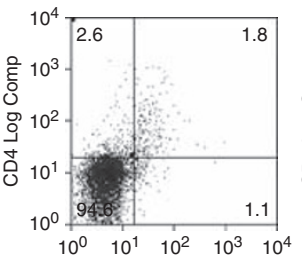

CD8 Log Comp

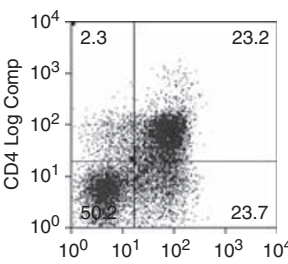

CD8 Log Comp

pMSCV-hCD2t infected HPCs

Figure 1 Reconstitution of FTOCs by thymocytes expressing glucocorticoid-induced genes. (a) Quantitation of apoptosis in murine thymocytes cultured in vitro \pm dexamethasone (Dex) \pm roscovitine (Rosc). The cells were permeabilized and intracellular DNA was stained with 7AAD. The percentages of sub-G $\mathrm{D}_{1} \mathrm{DNA}_{\mathrm{A}}$ content show the level of apoptosis in each sample. RNA isolated from thymocytes treated with Dex + Rosc and Rosc alone was processed for analysis on gene arrays. (b) List of genes most highly upregulated in dexamethasone-treated murine thymocytes as determined using Affymetrix MGu74Av2 gene arrays. (c) Time course of pMSCV-IREShCD2t retrovirally transduced HPCs reconstituting FTOCs. The upper panel shows hCD2 expression within FTOCs cultured for 7 and 14 days on filters and the lower panel shows CD4 and CD8 expression within the same FTOCs 
$\mathrm{hCD}^{+}$cells within the DP thymocyte population was quantified. The efficacy of the FTOC assay was tested by overexpression of the previously identified dexamethasone target genes Tdag8 (present in target list), Bax (absent in target list) and the anti-apoptotic gene $\mathrm{Bcl}-2$. HPCs were transduced, with between 70 and $90 \%$ efficiency, and hCD2 expression was measured pre- and post-FTOC. No change in the ratio of expressing cells was detected with the vector control (Figure 2a). A major drop in the ratio of both Tdag8and Bax-expressing cells was detected post-FTOC, confirming a negative effect on survival by both Tdag 8 and Bax on developing thymocytes (Figure 2a). The ratio of $B c /-2$-expressing cells went up from $90 \%$ to close to $100 \%$, confirming a pro-survival effect of $\mathrm{Bcl}-2$ on developing thymocytes. These data show that the FTOC assay can be used as a functional readout to assess the effect of retrovirally transduced genes on survival and T-cell development.

To assay their role in T-cell survival, HPCs were infected with retroviruses expressing candidate gene cDNAs and used to repopulate 2-deoxyguanosine (2dGuo)-depleted FTOCs in hanging drop culture. The FTOCs were transferred to filters for 13 days to determine the effect of candidate gene expression on thymocyte development and cell survival. Of the target genes assayed, Tdag8, Tnfaip8, A20 and E4bp4 were shown to confer a net negative effect on thymocyte survival in reconstituted FTOCs. These effects are shown for an extended set of genes using multiple FTOCs per gene $(n=4-6)$ in Figure $2 \mathrm{a}$. The percentage of $\mathrm{hCD}^{+}$cells representing the efficiency of HPC infection (pre-FTOC), the proportion of $\mathrm{hCD}^{+}$cells in reconstituted FTOCs following 13 days on filter (post-FTOC) and the proportion of DP cells in the reconstituted FTOCs, which are hCD2 ${ }^{+}$(post-FTOC DP) are shown. The pro-apoptotic effect of Tdag8, Tnfaip8 and E4bp4 within the DP population is significantly large (Figure 2a) and very similar in extent to Bax; however, mSpp1 has no effect on thymocyte survival and $A 20$ overexpression abrogates thymocyte development in this system.

We decided to focus our functional analysis on Tnfaip8 and Tdag8. We prepared reconstituted FTOCs using Tdag8 and Tnfaip8 to determine their effect on total cell number as well as the proportion of $\mathrm{hCD}^{+}$cells (Figure $2 \mathrm{~b}$ ). Both Tnfaip8 and Tdag8 had a profoundly negative effect on the survival of hCD2 ${ }^{+}$cells compared with total cells within the reconstituted FTOCs. This effect was identical to that caused by the pro-apoptotic gene Bax. a

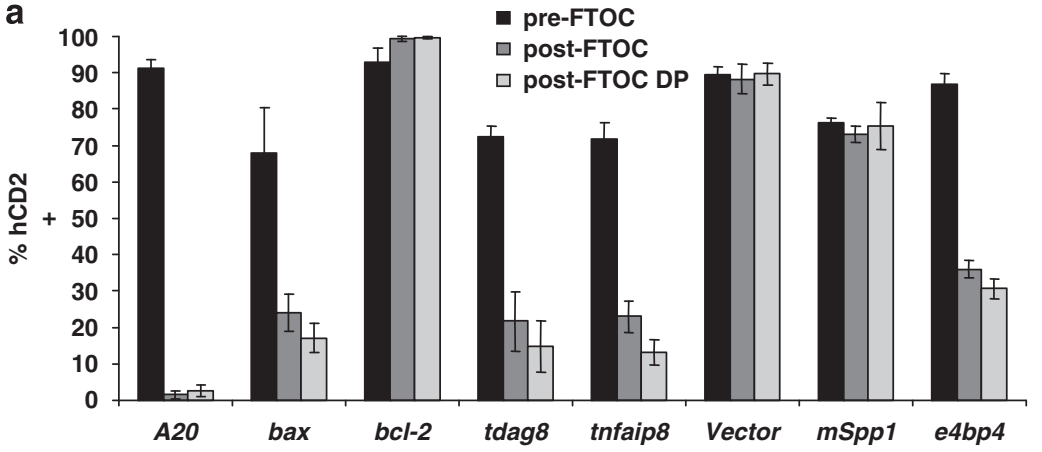

b

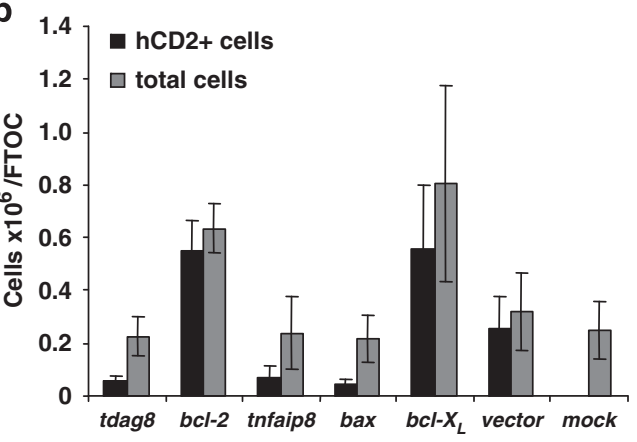

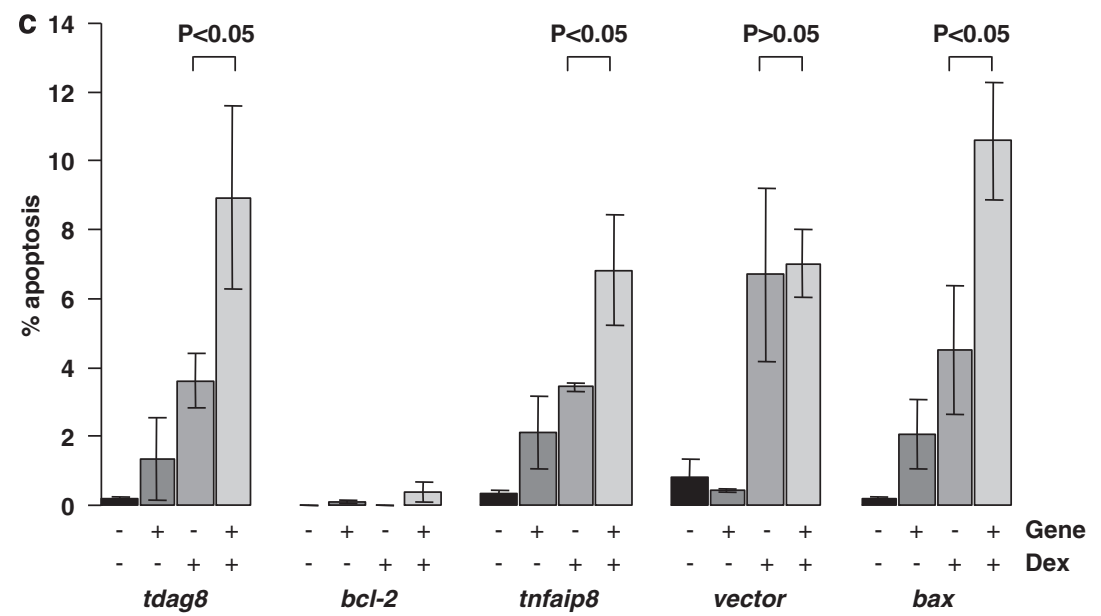

Figure 2 Candidate genes differentially affect thymocyte development and response to glucocorticoid within reconstituted FTOCs. (a) Percentage of hCD2 ${ }^{+}$cells for candidate genes using multiple FTOCs per gene $(n=4-6)$ are shown. Percentage of hCD2 ${ }^{+}$cells representing the efficiency of HPC infection (pre-FTOC), the proportion of hCD2 ${ }^{+}$cells in reconstituted FTOCs following 13 days on filter (post-FTOC) and the proportion of DP cells in the reconstituted FTOC which are hCD2 ${ }^{+}$(post-FTOC DP) are shown. (b) Multiple FTOCs $(n=4-5)$ reconstituted with HPCs transduced with thfaip8, tdag8 and control genes. Histogram shows total number of cells $\left(\times 10^{6}\right)$ per FTOC and total number of hCD2-expressing cells $\left(\times 10^{6}\right)$ per FTOC. (c) The level of apoptosis in thymocytes from reconstituted FTOCs following dexamethasone treatment was determined by flow cytometry $(n=4-6)$ and $P$-values shown were calculated for Dex alone versus Gene + Dex. Thymocytes were stained for CD8 and hCD2, saponinpermeabilized and stained with 7AAD to measure DNA content 
We determined the expression level of Tnfaip8 and Tdag8 during normal T-cell development by sorting thymocytes from normal thymuses into $\mathrm{CD}^{-} \mathrm{CD}^{-}$(DN); DP and CD4 or CD8 single positive (SP) cells, extracting RNA and performing quantitative PCR (Q-PCR). The major change observed was the dramatic upregulation of Tnfaip8 expression within the DP population (Supplementary Figure S2). To determine whether overexpression of Tnfaip8 and Tdag8 enhanced sensitivity to glucocorticoids, we exposed reconstituted FTOCs to $0.5 \mu \mathrm{M}$ dexamethasone for $4 \mathrm{~h}$. We had previously determined that $0.5 \mu \mathrm{M}$ dexamethasone sufficiently saturates the glucocorticoid receptor and induces a maximal apoptotic response (Supplementary Figure S3). It should be noted that the levels of apoptosis in thymocytes from intact FTOCs are in the range $0-10 \%$ as they were measured at early time points on small numbers of cells. Following dexamethasone treatment, the thymocytes were stained for CD8 and hCD2 cell surface expression, saponin-permeabilized and stained with 7AAD to measure DNA content. Significantly enhanced sensitivity to dexamethasone was found in $\mathrm{hCD}^{+}$thymocytes retrovirally infected with Tdag8, Tnfaip8 and the positive control, Bax (Figure 2c). It should be noted that the relevant comparison within each set of data points is the percentage of apoptosis following dexamethasone treatment in the presence or absence of the gene assayed. Therefore, overexpression of both Tdag8 and Tnfaip8 sensitizes thymocytes to glucocorticoid-induced apoptosis as previously shown for Bax. ${ }^{21}$

To confirm these effects on primary thymocytes in suspension using larger numbers of cells, we adapted the OP9-DL1 co-culture system ${ }^{22,23}$ to generate retrovirally transduced DP thymocytes. HPCs were retrovirally infected as before, but then $24 \mathrm{~h}$ post-infection they were used to seed six-well tissue culture dishes containing OP9-DL1 stromal cells. The cultures were split every 4-7 days and fresh IL-7 and Flt-3L was added. Figure 3 a shows a representative flow cytometric analysis of OP9-DL1-derived thymocytes 15 days after seeding and stained for CD4 and CD8 with gating on live hCD2-positive cells. DP thymocytes were produced in all cases; however, the apoptosis regulatory genes do not cause a developmental effect due to the presence of a high level of cell-protective cytokines such as IL-7. The OP9-DL1-derived thymocytes at day 15 post-seeding were treated with $0.5 \mu \mathrm{M}$ dexamethasone for $12 \mathrm{~h}$ to assess their response to glucocorticoid-mediated apoptosis. To determine the degree of apoptosis, the thymocytes were stained for CD8, hCD2 and DRAQ5 to determine DNA content (Figure 3b). The presence of the control anti-apoptotic genes $\mathrm{Bcl}-2$ and $\mathrm{Bcl}-x_{L}$ clearly protected the thymocytes against dexamethasone-induced apoptosis relative to vector only, whereas Bax promoted it. Tnfaip8 and Tdag8 both promoted apoptosis to a similar extent as Bax, confirming their potential as important regulators of glucocorticoid sensitivity in thymocytes.

Effect of RNAi-mediated knockdown of Tnfaip8 on glucocorticoid-induced apoptosis in FTOCs. Tdag8 and Tnfaip8 emerged from the initial screen and subsequent functional analysis as good candidate target genes for the regulation of glucocorticoid-mediated apoptosis. However, during the course of this work, Tdag $8^{-1-}$ mice had been generated and shown to have no defective survival response to glucocorticoid treatment, ${ }^{24}$ suggesting that there is redundancy in the function of Tdag8. Therefore, we decided to focus on Tnfaip 8 to determine if it has a crucial role in glucocorticoid-induced apoptosis. We used RNAi to selectively downregulate Tnfaip8 expression in FTOCs and monitor the effect of dexamethasone on thymocyte survival.

Predictive software was used to identify two shRNAs that would effectively knockdown Tnfaip8 expression (referred to as T8-RNAi-1 and T8-RNAi-2) directed against sequences corresponding to regions $\mathrm{N}$-terminal (amino acids 35-43) and C-terminal (amino acids 161-169) of the Tnfaip8 protein (Figure 4a). These sequences were screened for their effectiveness by assaying their ability to decrease $\beta$-galactosidase expression following co-transfection of 293T cells by a U6-promoter-driven shRNA expression vector and a vector expressing the LacZ-TNFAIP8 fusion protein. Both Tnfaip8 shRNAs achieved greater than $75 \%$ reduction in $\beta$-galactosidase levels compared with the control (Figure $4 \mathrm{~b}$ ). The two Tnfaip8 shRNAs were then cloned into a modified retroviral expression vector designated pMSCV-LTRmiR30-PIhCD (Supplementary Figure S1b), a modified form of a previously reported vector that embeds the shRNA sequence within a larger miRNA sequence so as to improve effectiveness. ${ }^{25}$ To determine the requirement for Tnfaip8 expression for glucocorticoid-mediated thymocyte apoptosis, HPCs were infected with shRNAmiR-expressing retroviruses and used to repopulate 2dGuo depleted FTOCs. The FTOCs were cultured for 13 days and then analyzed for the effect of the target gene knockdown compared with gene overexpression on thymocyte development. The retroviruses used had shRNAmiRs directed against Tnfaip8 and Bim (as a positive control) and an empty vector control. Flow cytometric analysis of hCD2, CD4 and CD8 staining showed that all the shRNAmiR retroviral expression vectors maintained expression throughout thymocyte development within FTOCs, did not affect development (Figure 4c) and demonstrated no loss or enrichment of hCD2 expression (Figure 4d). The repopulated FTOCs were then exposed to $0.5 \mu \mathrm{M}$ dexamethasone for $4 \mathrm{~h}$ and the thymocytes were stained for CD8 and hCD2, as well as DRAQ5, to determine DNA content. Thymocytes harboring the Bim shRNAmiR demonstrated significantly less apoptosis in response to dexamethasone than the vector control as expected from downregulating a gene known to be crucial to glucocorticoid-mediated apoptosis. ${ }^{12}$ FTOCs harboring both Tnfaip8 shRNAmiRs demonstrated comparable reductions to Bim in glucocorticoid-mediated thymocyte apoptosis relative to the control (Figure 4e). These data show that Tnfaip8 has a crucial role in glucocorticoid-induced thymocyte apoptosis.

Lymphocyte apoptosis induced by synthetic glucocorticoids is a critical component of the therapeutic regimes used to treat leukemia. ${ }^{26}$ Recent data have suggested that the glucocorticoid-induced apoptosis pathway utilized by leukemic cells may be more similar to that used by thymocytes than for normal mature T cells. ${ }^{27}$ Therefore, we tested the effect of knockdown of TNFAIP8 expression in the human leukemic cell line, CEM. ${ }^{28}$ CEM cells were transduced with retrovirus expressing shRNAmiRs directed against TNFAIP8 or a Control sequence and sorted on the basis of hCD2 expression. The stably transduced cells were exposed to increasing concentrations of dexamethasone and their viability was 

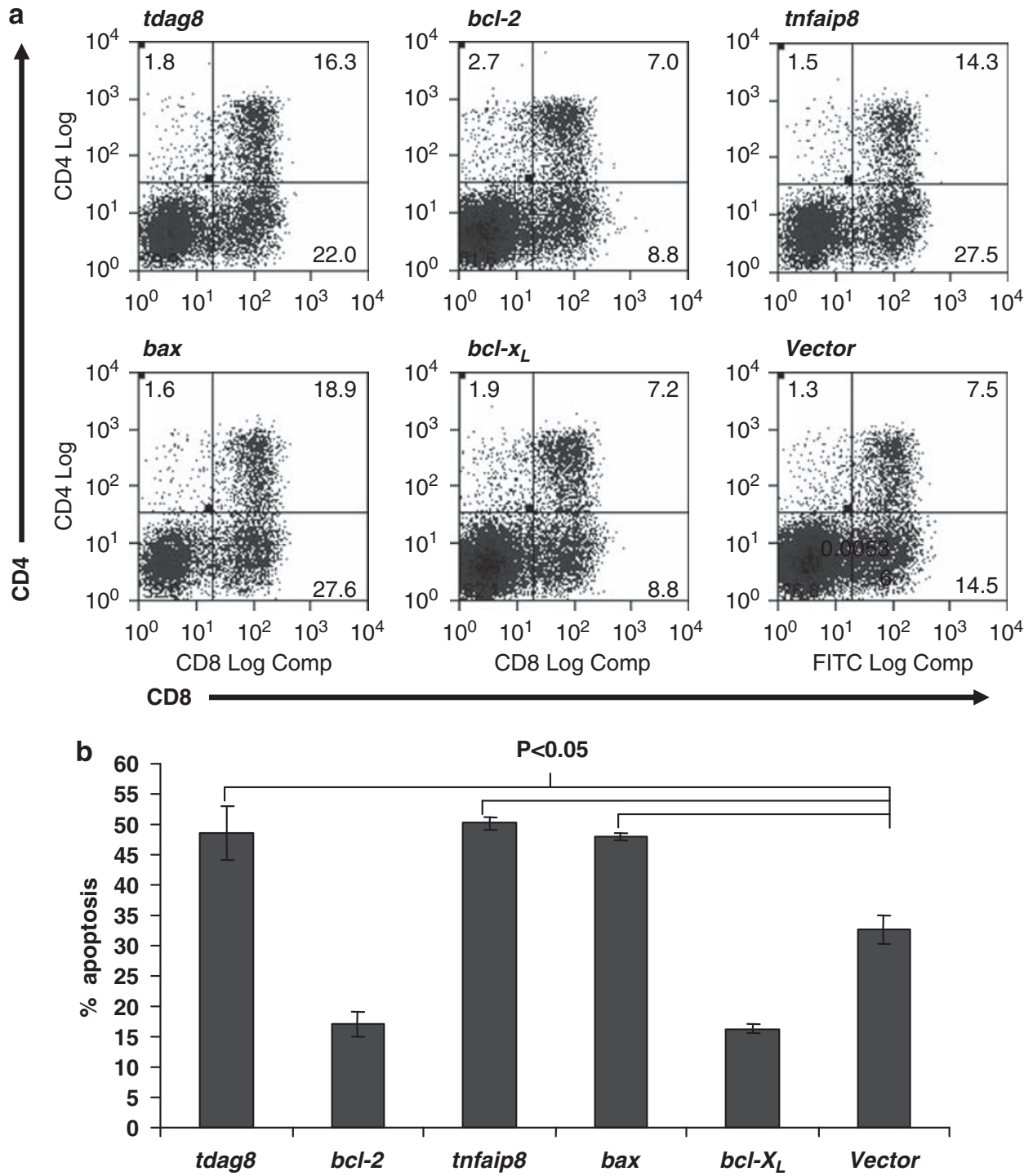

Figure 3 Action of Tnfaip8 and Tdag8 on OP9-DL1-derived thymocytes. (a) Thymocytes derived from 15-day OP9-DL1 co-culture of HPCs retrovirally transduced with individual genes, stained for CD4 and CD8 gated on live, $\mathrm{hCD} 2^{+}$cells. (b) Determination of level of apoptosis in OP9-DL1-derived thymocytes transduced with individual genes and treated with $0.5 \mu \mathrm{M}$ dexamethasone for $12 \mathrm{~h}$ ( $n=4-6$ per gene and $P<0.05$ in each case). The thymocytes were stained for CD8, $h C D 2$ and DRAQ5 to determine DNA content and hence percentage of cells undergoing apoptosis

assessed after 18h. CEM cells harboring both TNFAIP8directed shRNAmiRs showed a pro-survival effect compared with the controls at increasing doses of dexamethasone (Supplementary Figure S4), which is entirely consistent with the protective effect seen on knocking down Tnfaip8 in FTOCs.

\section{Discussion}

Although glucocorticoid-mediated apoptosis of thymocytes was first described over a quarter of a century ago, its mechanistic basis remains unclear as does its role in T-cell selection and immune homeostasis. Glucocorticoids have been hypothesized to have multiple roles from directing selection processes in $\mathrm{T}$-cell maturation ${ }^{1}$ and molding the $\mathrm{T}$-cell repertoire ${ }^{2}$ to regulating thymocyte apoptosis following polyclonal T-cell activation. ${ }^{29}$ However, the central requirement for de novo gene expression in glucocorticoidmediated apoptosis of thymocytes is clearly established. ${ }^{4,5}$

Using DNA microarrays, we identified genes upregulated in primary thymocytes on exposure to the glucocorticoid, dexamethasone. We then tested these genes for proapoptotic actions in primary thymocytes both in FTOCs and in thymocytes derived from HPCs grown on OP9-DL1 stromal cells. This effectively allowed us to identify genes that could act on thymocyte survival in response to a specific single proapoptotic stimulus. By then knocking down Tnfaip8 expression in individual FTOCs, we were able to show that this gene is critical for glucocorticoid-mediated thymocyte apoptosis. Bim is the only other gene characterized to have a critical role in thymocyte apoptosis driven by glucocorticoids. ${ }^{11}$ By analogy with Bim, we propose that Tnfaip8 is critical to the proapoptotic response of thymocytes to glucocorticoids. The 
a

\begin{tabular}{|cccc|}
\multicolumn{4}{c}{$\begin{array}{c}\text { Tnfaip8 } \\
109\end{array}$} \\
\hline & & 198 & \\
\hline & Putative DED & & \\
\hline $35-43$ & & & $161-169$
\end{tabular}

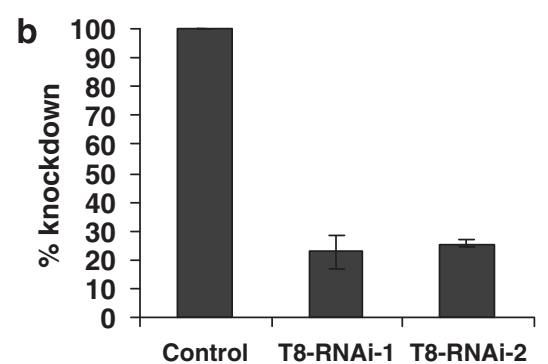

C
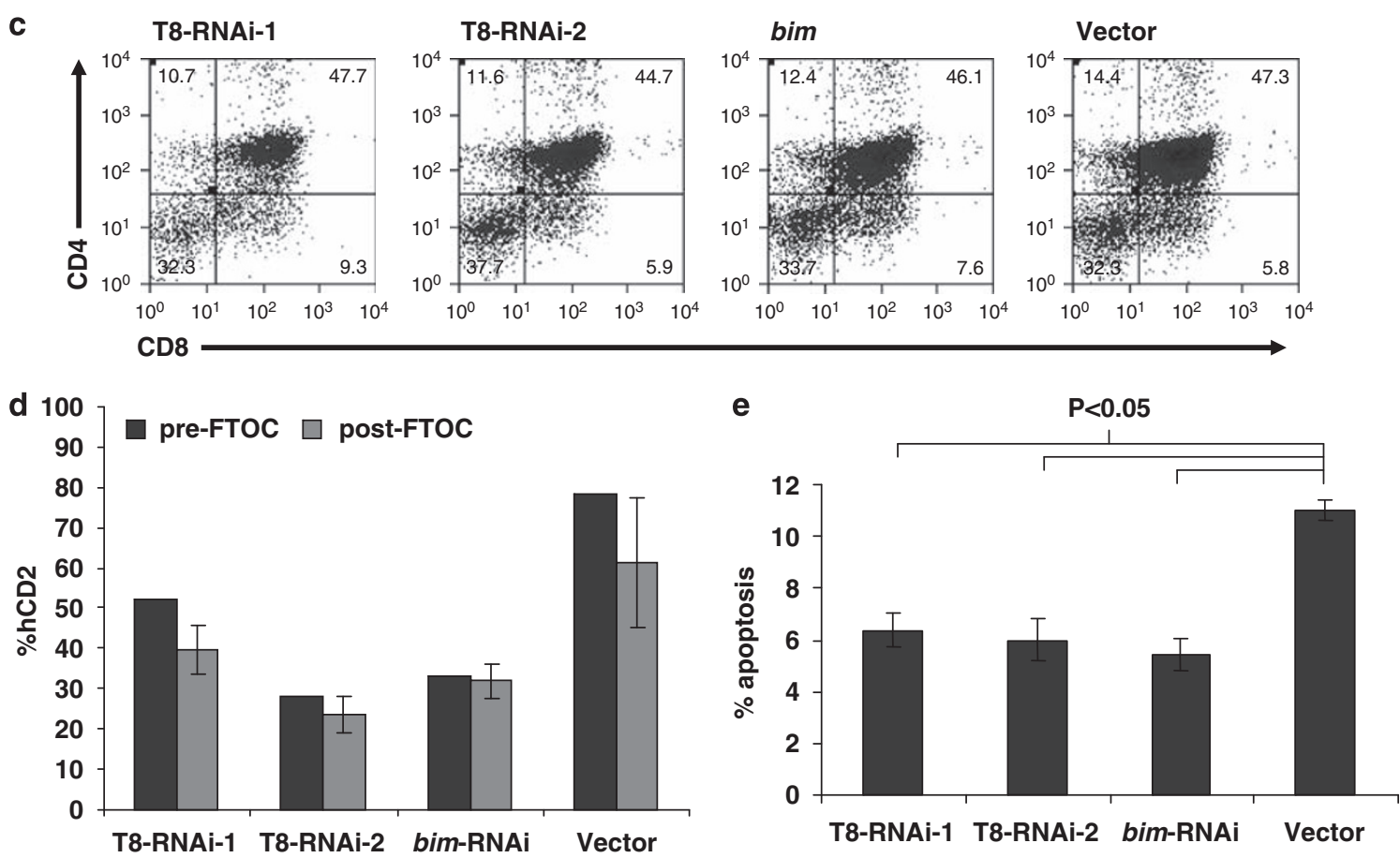

Figure 4 RNAi-mediated knockdown of Tnfaip8 in FTOCs and the effect on glucocorticoid-mediated apoptosis. (a) Schematic diagram of the protein structure of Tnfaip8 highlighting the putative DED. Two shRNAmiRs identified by predictive software (T8-RNAi-1 and T8-RNAi-2) directed against the regions of Tnfaip8 indicated by the thick lines underneath the protein structure. (b) Percentage knockdown of LacZ-TNFAIP8 fusion protein in 293T cells transfected with vector expressing each shRNA relative to mocktransfected control. (c) Flow cytometry of 13-day thymocytes from FTOCs reconstituted with HPCs transduced by T8-RNAi-1, T8-RNAi-2, Bim and vector control shRNAexpressing retroviruses, stained for CD4 and CD8 and gated for hCD2-expressing cells. (d) Percentage CD2-expressing cells in FTOCs ( $n=4-6)$ reconstituted with HPCs transduced with shRNAmiRs directed against Tnfaip8 or Bim, and a vector-only transduced control. FTOCs were stained for hCD2, CD4 and CD8 and analyzed by flow cytometry. (e) Percentage apoptosis in the repopulated FTOCs treated with $0.5 \mu \mathrm{M}$ dexamethasone for $4 \mathrm{~h}$ ( $n=4-6$ and $P<0.05$ in each condition). Thymocytes were stained for CD8, hCD2 and DRAQ5 to determine DNA content as a measure of the level of apoptosis

function of TNFAIP8 protein is not known, but it contains a death effector domain (DED) and can block CASPASEmediated apoptosis. ${ }^{30}$ Based on sequence homology, a TNFAIP8 family of proteins has very recently been defined consisting of TNFAIP8, TIPE1 (TNFAIP8Like1), TIPE2 and TIPE3. $^{31}$ The TNFAIP8 family members do not share significant sequence homology with other proteins apart from limited homology within the DED. However, a dramatic breakthrough in identifying their role has come with the generation of Tipe $^{-1-}$ mice. ${ }^{31}$ The Tipe $2^{-/-}$mice exhibit inflammation in many organs, splenomegaly and premature death with defective innate and adaptive immune processes. TNFAIP8 may be the molecular link between glucocorticoidmediated cell death and immune homeostasis. ${ }^{3}$

The experimental approach that we have developed to manipulate gene expression in reconstituted FTOCs has wide application in the studies of T-cell function particularly for studies of cell survival or response to apoptotic stimuli. It is worth noting that in order to identify Tnfaip8 as crucial we used both overexpression and downregulation of expression. It is essential to do both as overexpression of Tdag8 in thymocytes suggested that it may have an essential role in thymocyte apoptosis; ${ }^{18}$ however, thymocytes from Tdag $^{-1-}$ mice have no enhanced protection against glucocorticoidmediated cell death. ${ }^{24}$

\section{Materials and Methods}

Microarray analysis. RNA was extracted in Trizol according to the manufacturer's instructions and further purified over RNeasy mini columns (Qiagen, Crawley, UK), and then checked for integrity, concentration and genomic DNA contamination using an Agilent (Palo Alto, CA, USA) 2100 Bioanalyser. RNA templates $(10 \mu \mathrm{g})$ were used for the multistep synthesis of biotin- 
labeled cRNA, which was then hybridized to Affymetrix GeneChip probe arrays according to protocols outlined in the Affymetrix Gene Expression Technical Manual; experiments were performed in triplicate. Data were analyzed using Affymetrix Microarray Suite version 5.0, with normalization performed using GeneSpring 5.0 to allow comparison across panels of multiple arrays. Transcripts scored as detected in $\geqslant 2$ arrays per triplicate; and exhibiting an $\geqslant 1.5$-fold difference in RNA levels compared with the controls were considered to be regulated. Statistical $t$-testing was used to confirm differentially regulated transcripts, and Benjamini-Hochberg multiple testing corrections was used to reduce the incidence of false positives.

Q-PCR. Taqman real-time Q-PCR assays were performed according to the manufacturer's instructions (Applied Biosystems, Foster City, CA, USA). Primers and probes were designed using Primer Express (Applied Biosystems), sequences available on request. Mus musculus poly(A)-specific ribonuclease (deadenylation nuclease) (PARN), was identified from microarray data across several different parallel Affymetrix GeneChip probe array screens as a gene whose expression does not vary during thymocyte apoptosis and was used as a loading control. HPRT was also used as an additional loading control.

Primary thymocytes and FTOCs. Primary thymocytes were isolated and cultured as described previously. ${ }^{21}$ FTOCs were prepared as described previously $\mathrm{y}^{32}$ and depopulated in the presence of $1.5 \mathrm{mM} 2 \mathrm{dGuo}$ in six-well tissue culture dishes. After 5-day depopulation, FTOC filters were moved to media without $2 \mathrm{dGuo}{ }^{33}$ for 1 day and then each FTOC lobe was reconstituted with $1-3 \times 10^{4}$ retrovirally infected ( $24 \mathrm{~h}$ post-infection) $\mathrm{CKit}^{+} \mathrm{HPCs}$ in a well of a Terasaki plate. After $24 \mathrm{~h}$, the reconstituted FTOCs were transferred to a fresh filter and left to culture at $37^{\circ} \mathrm{C}$ for $7-17$ days with one media change after $7-10$ days.

OP9-DL1 co-cultures. OP9-DL1 stromal cells were maintained as described. ${ }^{22}$ For co-cultures, OP9-DL1 cells were split 1:5 into six-well plates 2 days before co-culture initiation, achieving $\approx 75 \%$ confluence. OP9-DL1 cells were washed and fresh medium was added with $1 \mathrm{ng} / \mathrm{ml} \mathrm{IL-7} \mathrm{(Peprotech)} \mathrm{and} 5 \mathrm{ng} / \mathrm{ml} \mathrm{Flt-3}$ ligand (Peprotech). Each well was initially inoculated with $1 \times 10^{4} \mathrm{HPCs}$ at $24 \mathrm{~h}$ postretroviral infection. Co-cultures were incubated for 7 days with one change of medium at 4 days. After 7 days, the developing thymocytes were placed on fresh OP9-DL1 cells at a $1: 10$ split. Further $1: 4$ splits were performed at 12,16 and 20 days.

Retroviral transduction of cells. $\mathrm{C}-\mathrm{Kit}^{+} \mathrm{HPC}$ were enriched using anti-cKit-biotin (2B8; BD Biosciences, San Jose, CA, USA) from the fetal liver of stage embryonic day E13.5 of C57BL/6 mouse embryos by magnetic cell sorting (Miltenyi Biotec, Cologne, Germany). Retroviral transduction was performed as described previously. ${ }^{34}$ CEM cells (CCRF-CEM obtained from HPACC, Salisbury, UK) were cultured in DMEM 10\% FCS. Retrovirus was produced from the Platinum-GP packaging cell line (Cell Biolabs, San Diego, CA, USA) co-transfected with VSV-G and shRNAmiR expression vectors.

Retroviral vectors and RNAi. To facilitate long-term expression of transduced genes in HPCs and derived thymocytes, the pMSCV-neo (Clontech, Mountain View, CA, USA) was modified. The phosphoglycerate kinase promoter and neomycin resistance cassette was replaced by an internal ribosome entry site (IRES) and truncated human CD2 (hCD2t) cassette amplified by PCR from the pMIIRES-hCD2 vector (gift from Dr. MJ Bevan (Department of Immunology, University of Washington, Seattle, WA, USA). The pMSCV-LTRmiR30-PIG (LMP) RNAi vector (Open Biosystems, Huntsville, AL, USA) was adapted to express the truncated hCD2 instead of EGFP. The shRNAmiR oligo hairpins (Sigma-Genosys, Dorset, UK) were originally designed on the Invitrogen (Paisley, UK) RNAiCentral website. Purified oligos were annealed to form and then ligated into Xhol/EcoRI-digested pMSCVLTRmiR30-PIhCD2 and the constructs were confirmed by DNA sequencing. For CEM cell transductions, the same expression vector was used containing shRNA sequences against TNFAIP8 (TNFAIP8.1: CTGCGTGCGTTTCAGTGTTTAA and TNFAIP8.2: CGGTCTTGATATTGAGATAATA) as well as a validated non-silencing Control sequence (RHS1707; Open Biosystems), cloned as above. Following transduction, CEM cells were sorted using anti-CD2 microbeads (Miltenyi Biotec) and cultured at a density of $5 \times 10^{4}$ cells per well in flat-bottomed, 96-well plates with various concentrations of dexamethasone (Sigma, Dorset, UK). After $24 \mathrm{~h}$, CellTiter 96 Aqueous One Solution Reagent (Promega, Madison, WI, USA) was added to each well and, following $4 \mathrm{~h}$ in culture, and cell viability was determined by measuring the absorbance at $490 \mathrm{~nm}$ using a plate-reader (Bio-Rad, Hercules, CA, USA). Each data point was measured in triplicate.

Flow cytometric analysis. Cells were pre-incubated with unlabeled anti-Fc $\gamma$ III/II receptor mAb2.4G2 diluted in FACS staining buffer (PBS containing 0.1\% BSA and $0.1 \%$ sodium azide). Cells were stained with CD4 (RMA4-5 or RPA-T4), CD8 (53-6.7), c-Kit (2B8) and human CD2 (RPA-2.10).

For intracellular DNA staining with 7AAD, cells were permeabilized with $0.03 \%$ saponin following cell surface staining, incubating with $0.03 \%$ saponin (Sigma) and $2.5 \mu \mathrm{g} / \mathrm{ml}$ 7AAD (Sigma) in FACS staining buffer at $37^{\circ} \mathrm{C}$ for $30 \mathrm{~min}$, and then stored until analysis at $4^{\circ} \mathrm{C}$. For DRAQ5 staining, cells were first stained for cell-surface markers and then resuspended in $300 \mu \mathrm{l}$ FACS staining buffer containing $20 \mu \mathrm{M}$ DRAQ5.

Acknowledgements. We thank Neil Thomas for help with initial Q-PCRs. This work was primarily supported by the Medical Research Council (UK) through Career Establishment Grant G9900172 (to HJMB). Additional funding was provided by Children with Leukaemia and the REACH Leukaemia Appeal.

\section{Conflict of interest}

The authors declare no conflict of interest.

1. Ashwell JD, Lu FW, Vacchio MS. Glucocorticoids in T cell development and function. Ann Rev Immunol 2000; 18: 309-345.

2. Van Laetham F, Baus E, Smyth LA, Andris F, Bex F, Urbain J et al. Glucocorticoids attenuate T cell receptor signaling. J Exp Med 2001; 193: 803-814.

3. Jondal M, Pazirandeh A, Okret S. Different roles for glucocorticoids in thymocyte homeostasis? Trends Immunol 2004; 25: 595-600.

4. Wyllie AH, Morris RG, Smith AL, Dunlop D. Chromatin cleavage in apoptosis: association with condensed chromatin morphology and dependence on macromolecular synthesis. $J$ Pathol 1984; 42: 67-77.

5. Cohen JJ, Duke RC. Glucocorticoid activation of a calcium-dependent endonuclease in thymocyte nuclei leads to cell death. J Immunol 1984; 132: 38-42.

6. McConkey DJ, Hartzell P, Orrenius S. Rapid turnover of endogenous endonuclease activity in thymocytes: effects of inhibitors of macromolecular synthesis. Arch Biochem Biophys 1990; 278: 284-287.

7. Lépine S, Sulpice JC, Giraud F. Signaling pathways involved in glucocorticoid-induced apoptosis of thymocytes. Crit Rev Immunol 2005; 25: 263-288.

8. Rathmell JC, Lindsten T, Zong WX, Cinalli RM, Thompson CB. Deficiency in Bak and Bax perturbs thymic selection and lymphoid homeostasis. Nat Immunol 2002; 3: 932-939.

9. Wei MC, Zong WX, Cheng EH, Lindsten T, Panoutsakopoulou V, Ross AJ et al. Proapoptotic BAX and BAK: a requisite gateway to mitochondrial dysfunction and death. Science 2001; 292: 727-730.

10. Wang Z, Malone MH, $\mathrm{He} \mathrm{H}, \mathrm{McColl} \mathrm{KS}$, Distelhorst $\mathrm{CW}$. Microarray analysis uncovers the induction of the proapoptotic BH3-only protein Bim in multiple models of glucocorticoidinduced apoptosis. J Biol Chem 2003; 278: 23861-23867.

11. Bouillet P, Metcalf D, Huang DCS, Tarlinton DM, Kay TWH, Kontgen F et al. Proapoptotic Bcl-2 relative Bim required for certain apoptotic responses, leukocyte homeostasis, and to preclude autoimmunity. Science 1999; 286: 1735-1738.

12. Abrams MT, Robertson NM, Yoon K, Wickstrom E. Inhibition of glucocorticoid-induced apoptosis by targeting the major splice variants of BIM mRNA with small interfering RNA and short hairpin RNA. J Biol Chem 2004; 279: 55809-55817.

13. Ashwell JD, Vacchio MS, Galon J. Do glucocorticoids participate in thymocyte development? Immunol Today 2000; 21: 644-646.

14. Godfrey DI, Purton JF, Boyd RL, Cole TJ. Glucocorticoids and the thymus: the view from the middle of the road. Trends Immunol 2001; 22: 243.

15. Pazirandeh $\mathrm{A}, \mathrm{Xue} \mathrm{Y}$, Prestegaard $\mathrm{T}$, Jondal M, Okret S. Effects of altered glucocorticoid sensitivity in the $T$ cell lineage on thymocyte and T cell homeostasis. FASEB J 2002; 16: 727-729.

16. Pazirandeh $\mathrm{A}$, Jondal M, Okret $\mathrm{S}$. Conditional expression of a glucocorticoid receptor transgene in thymocytes reveals a role for thymic-derived glucocorticoids in thymopoiesis in vivo. Endocrinology 2005; 146: 2501-2507.

17. Granés F, Roig MB, Brady HJM, Gil-Gómez G. Cdk2 activation acts upstream of the mitochondrion during glucocorticoid induced thymocyte apoptosis. Eur J Immunol 2004; 34: $2781-2790$.

18. Tosa N, Murakami M, Jia WY, Yokoyama M, Masunaga T, Iwabuchi C et al. Critical function of $\mathrm{T}$ cell death-associated gene 8 in glucocorticoid-induced thymocyte apoptosis. Int Immunol 2003; 15: 741-749.

19. Malone MH, Wang Z, Distelhorst CW. The glucocorticoid-induced gene tdag8 encodes a pro-apoptotic $G$ protein-coupled receptor whose activation promotes glucocorticoidinduced apoptosis. J Biol Chem 2004; 279: 52850-52859.

20. Wang Z, Malone MH, Thomenius MJ, Zhong F, Xu F, Distelhorst CW. Dexamethasoneinduced gene 2 (dig2) is a novel pro-survival stress gene induced rapidly by diverse apoptotic signals. J Biol Chem 2003; 278: 27053-27058. 
21. Brady HJM, Salomons GS, Bobeldijk RC, Berns AJ. T cells from baxalpha transgenic mice show accelerated apoptosis in response to stimuli but do not show restored DNA damageinduced cell death in the absence of p53. EMBO J 1996; 15: 1221-1230.

22. Schmitt TM, Zúñiga-Pflücker JC. Induction of T-cell development from hematopoietic progenitor cells by delta-like-1 in vitro. Immunity 2002; 17: 749-756.

23. Zúñiga-Pflücker JC. T-cell development made simple. Nat Rev Immunol 2004; 4: 67-72.

24. Radu CG, Cheng D, Nijagal A, Riedinger M, McLaughlin J, Yang LV et al. Normal immune development and glucocorticoid-induced thymocyte apoptosis in mice deficient for the T-cell death-associated gene 8 receptor. Mol Cell Biol 2006; 26: 668-677.

25. Dickins RA, Hemann MT, Zilfou JT, Simpson DR, Ibarra I, Hannon GJ et al. Probing tumor phenotypes using stable and regulated synthetic microRNA precursors. Nat Genet 2005; 37: $1289-1295$.

26. Frankfurt O, Rosen ST. Mechanisms of glucocorticoid-induced apoptosis in hematologic malignancies: updates. Curr Opin Oncol 2004; 16: 553-563.

27. Wang D, Müller N, McPherson KG, Reichardt HM. Glucocorticoids engage different signal transduction pathways to induce apoptosis in thymocytes and mature $\mathrm{T}$ cells. $\mathrm{J}$ Immunol 2006; 176: 1695-1702.

28. Bansal N, Houle A, Melnykovych G. Apoptosis: mode of cell death induced in T cell leukemia lines by dexamethasone and other agents. FASEB J 1991; 5: 211-216.
29. Baumann S, Dostert A, Novac N, Bauer A, Schmid W, Fas SC et al. Glucocorticoids inhibit activation-induced cell death (AICD) via direct DNA-dependent repression of the CD95 ligand gene by a glucocorticoid receptor dimer. Blood 2005; 106: $617-625$.

30. Kumar D, Whiteside TL, Kasid U. Identification of a novel tumor necrosis factor-alphainducible gene, SCC-S2, containing the consensus sequence of a death effector domain of fas-associated death domain-like interleukin-1beta-converting enzyme-inhibitory protein. J Biol Chem 2000; 275: 2973-2978.

31. Sun H, Gong S, Carmody RJ, Hilliard A, Li L, Sun J et al. TIPE2, a negative regulator of innate and adaptive immunity that maintains immune homeostasis. Cell 2008; 133 : 415-426.

32. Williams $\mathrm{O}$, Norton T, Halligey M, Kioussis D, Brady HJM. The action of Bax and Bcl-2 on T cell selection. J Exp Med 1998; 188: 1125-1133.

33. Jenkinson EJ, Franchi LL, Kingston R, Owen JJ. Effect of deoxyguanosine on lymphopoiesis in the developing thymus rudiment in vitro: application in the production of chimeric thymus rudiments. Eur J Immunol 1982; 12: 583-587.

34. Morrow M, Horton S, Kioussis D, Brady HJM, Williams O. TEL-AML1 promotes development of specific hematopoietic lineages consistent with pre-leukemic activity. Blood 2004; 103: 3890-3896.

Supplementary Information accompanies the paper on Cell Death and Differentiation website (http://www.nature.com/cdd) 